We appreciate the response to this publication feature and welcome all contributions. Contributions may be sent to José A. Mascorro, our Technical Editor, at hise-mail address: jmascor@tulane.edu. José may also be reached at the Department of Structural and Cellular Biology, Tulane University Health Sciences Center, 1430 Tulane Ave., New Orleans, LA 70112 and $P h:$ (504) 584-2747 (504) 584-1687

\section{New Resin for Repair of Bell Jar Chips}

Owen P. Mills and Matt Huuki*

Michigan Technological University, ${ }^{*}$ Matt's Auto Glass, Houghton, MI opmills@mtu.edu and mhuuki@global.net

As you ease the bell jar back down onto its seat, past the evaporation fixture, it is quite easy to chip a $\$ 600$ jar. Chipped bell jars are a perennial problem in EM labs. They degrade vacuum in evaporators resulting in lower quality films and ultimately shorten the life of the vacuum
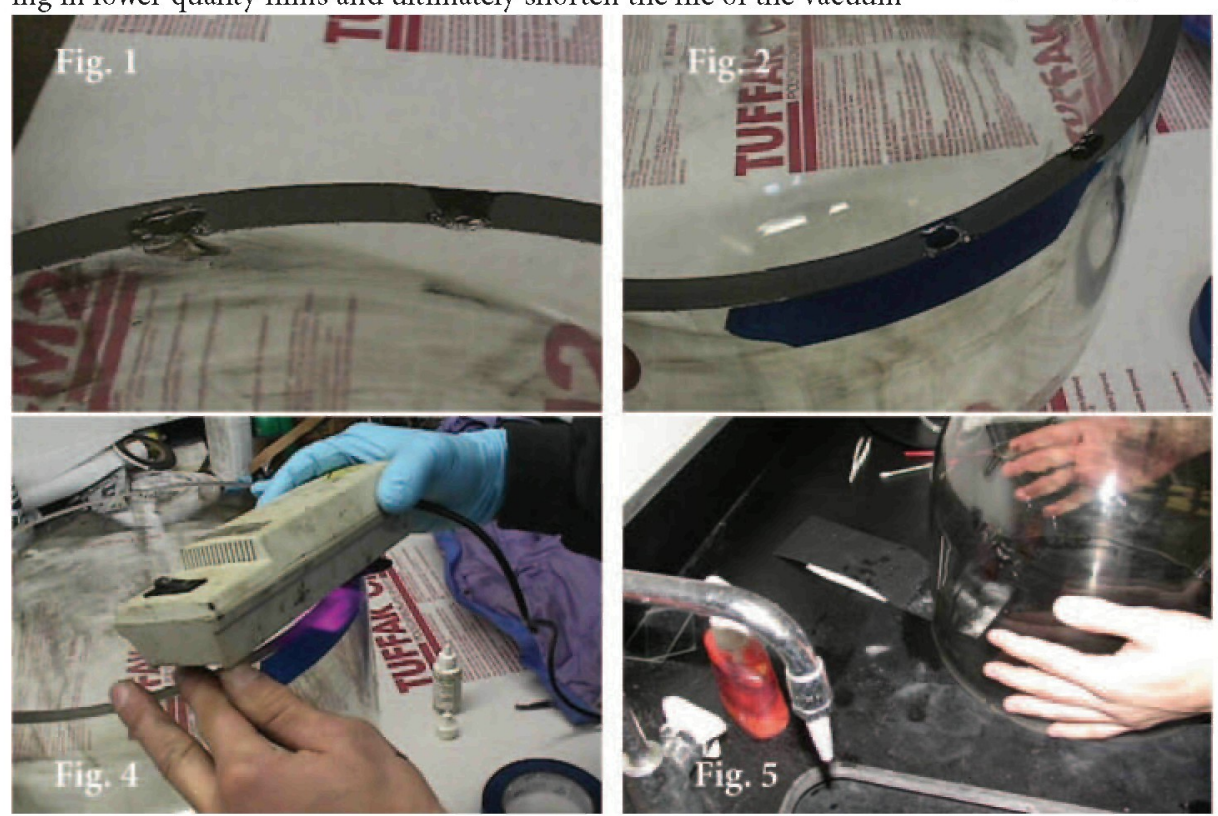

stuck down to a lab bench top. I wet the paper and slid the bell jar back and forth across the wet sandpaper, taking care to keep the bell jar flat against the bench top surface at all times (Fig. 5). The finished repair is shown in Fig. 6.

This is the second jar I have repaired using this technique. Both repaired bell jars hold vacuum and have performed well in all ways. I would recommend this technique to those with shallow chips of small extent. Be aware that your local auto glass repair shop will have no idea what a bell jar is, so be prepared to explain your situation. Better yet, bring this copy of MT with you!

$\uparrow$ Please read the Microscopy Today Disclaimer on page 6. This procedure is potentially dangerous. DO NOT ATTEMPT this repair on ANY size bell jar defect without an understanding of the risks involved and your commitment to taking ALL appropriate precautions during the repair process and after the repaired bell jar is returned to service. If you are unsure of the consequences of your actions, don't do it!

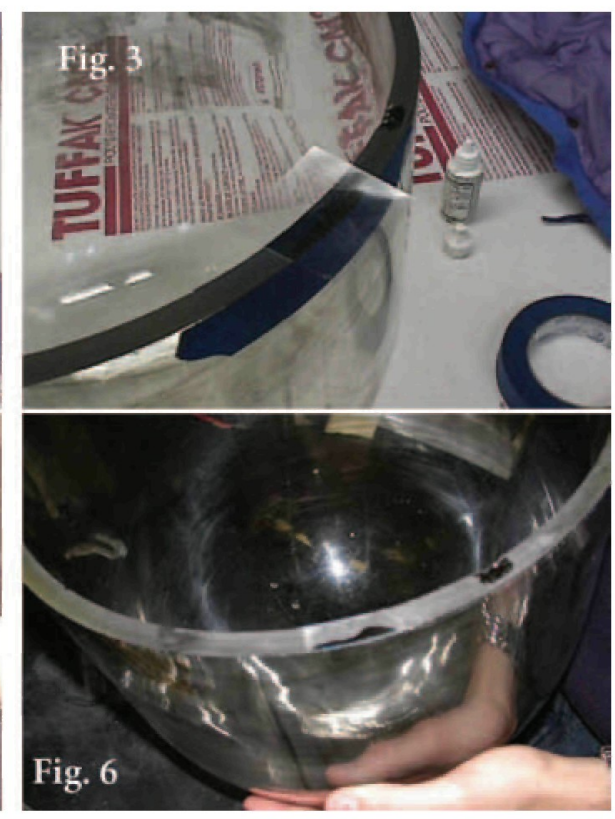

Inexpensive Digitization of an SEM

system. This note describes a new resin that I have used for repairing bell jar chips.

A common chip can be seen in Fig. 1. It was caused by crashing the bell jar into the evaporation fixture. The chip is less than $0.5 \mathrm{~mm}$ deep. Beside it you can see another chip that will not be treated as it does not extend to the jar edge.

IMPORTANT NOTE TO READERS: It would not be wise to attempt repairs on deep chips of $\geq 0.10 \mathrm{~mm}$ or those spreading over $30 \mathrm{~mm}$ laterally. Finally, DO NOT attempt repairs on cracked bell jars. Bell jars must resist the stress of vacuum and cracked bell jars must be taken out of service. $\dagger$

I worked with Matt Huuki, owner of Matt's Auto Glass in Houghton, MI, to repair this bell jar chip. The resin Matt uses is Glas-Weld \#2020 Clear Resin. This is the same product he uses to repair rock chips in automotive windshields. It is a UV sensitive resin that sets in an anaerobic environment.

We made the repair as shown in the follow sequence of photos. In Fig. 2 a dam of masking tape is applied to both sides of the jar to contain the thin epoxy. Slightly more resin is applied than needed and a mylar sheet is set over the wet resin in Fig. 3. The UV light is then applied for $\sim 10$ minutes (Fig. 4). The UV light is applied for a longer period of time for this bell jar repair since the resin is thicker compared with a windshield chip repair where the resin is normally very thin.

After the UV is applied, the resin is dry and the tape can be removed. Next, the resin can be ground down to the same height as the ground edge of the bell jar. To accomplish this I used 240 grit SiC pressure sensitive adhesive (PSA) backed wet-dry sandpaper that we temporarily
Henry C. Aldrich and Donna S. Williams, University of Florida, Gainesville, FL haldrich2@cox.net

Because of the high cost of Polaroid film, many years ago we fitted our Hitachi S-450 scanning electron microscope with a $35 \mathrm{~mm}$ camera. At that time, we used a Pentax ME Super, which was totally manual and had to have the film advanced by a hand lever. This was an annoyance, but when we set up the system, Polaroid Type 55 film was about $\$ 2.00$ per photo, and the cost of $35 \mathrm{~mm}$ spooled in our lab ran about $\$ .10$ per photo.

When we traded the Hitachi S-450 for the later Hitachi S-570, we moved the $35 \mathrm{~mm}$ system to this microscope. About 1999, when the Pentax ZX-50 with motorized film advance became available, we adapted it to the S-570, using the Pentax electric shutter release. The lens used with both of these cameras was an elderly $50 \mathrm{~mm}$ screw-mount Pentax Macro lens that focused well on the CRT of the SEM.

Then, when Canon introduced the Digital Rebel for under $\$ 1000$ about a year ago, we convinced a local Canon dealer to lend us one to try on the SEM. It worked quite well, and so we purchased it and converted the Hitachi S-570 to digital photography for about $\$ 1000$ - the cost of the Digital Rebel with its standard zoom lens plus the electric cable release. We also purchased an AC adapter to replace the AA batteries. The Rebel uses Compact Flash cards, which can then be removed from the camera and taken to any computer with a card reader to download the images. We have not experimented with other lenses. A dedicated macro lens might produce slightly sharper images, but the standard Rebel zoom lens seems quite adequate. The Canon electric cable release has a locking feature, so 


\section{IIIOROSOOPY 101}

when the camera is in manual exposure mode set to "Bulb", the shutter stays open as long as the lock is down. We use the 100 second exposure setting on the Hitachi SEM and $\mathrm{f}-16$ on the Canon lens. Some CCD chips tend to become noisy at longer exposure times, but the Canon has a CMOS chip that does not seem prone to this difficulty. It was surprising to learn that not all digital cameras support longer shutter speeds on the "B" setting. One digital camera (not an SLR), that we initially tried would go no longer than 50 seconds and had a noisy CCD chip. It is necessary to have an $\mathrm{AC}$ power supply, because the battery is being drained as long as the shutter is open at the " $\mathrm{B}$ " setting.

As this is written, the 6.3 megapixel Canon EOS Digital Rebel (apparently also known as the 300D) is available for $\$ 854.95$ (late 2004) after rebate from New York discount houses, including the $18-55 \mathrm{~mm}$ lens and a one gigabyte memory card. The electric release is $\$ 25.95$ and the AC adapter $\$ 64.95$. The camera will record both RAW and JPEG files. We have compared the two modes and see little or no advantage to using the RAW file mode for this application, although we find RAW very useful for normal color work. Ordinarily we discard the color information as soon as we download the JPEG file into the computer using Photoshop, and then archive the grayscale images on a hard drive in TIFF format to avoid loss of image quality.

Figure A shows the camera on its mount, which was improvised as an extension of the bracket at left that originally held the Polaroid camera. We simply added a U-shaped piece of aluminum with a slot in it for a tripod sized screw to hold the Canon camera, and fixed this aluminum bracket to the housing with the screw shown at left. The CRT is indicated by the white arrow. Figure B shows the operating arrangement, with a cardboard box covering the camera to exclude room light and dust. Black arrow shows the electric shutter release for the camera. Figure $\mathrm{C}$ shows part of a bacterial biofilm photographed with the $35 \mathrm{~mm}$ camera. Figure D shows the same specimen photographed with the Canon digital Rebel as described herein.
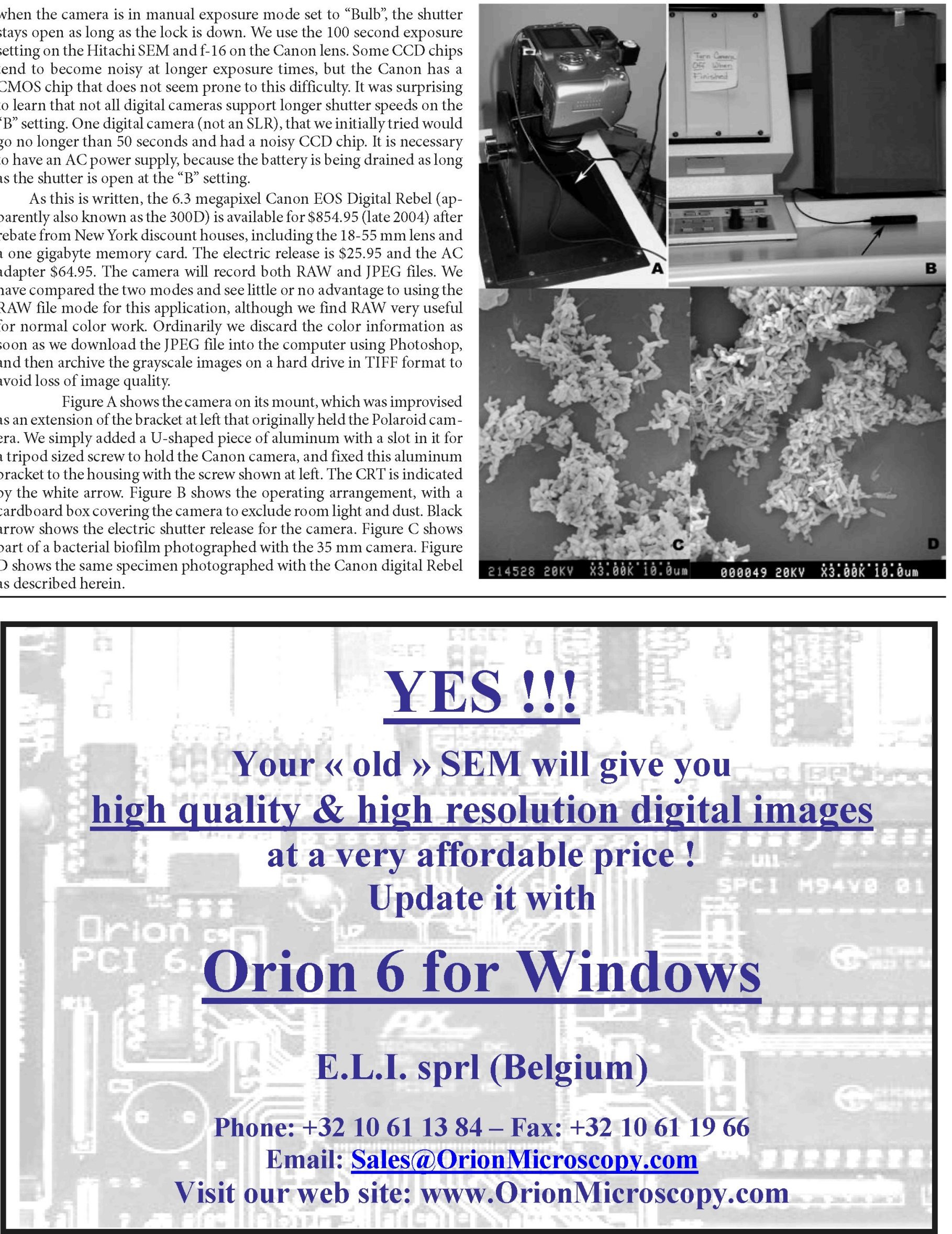